VNU Journal of Science: Natural Sciences and Technology Journal homepage: https://js.vnu.edu.vn/NST

Original Article

\title{
Assessment of Gas-Particle Phase Distribution and Source Apportionment of Atmospheric Phthalate Esters and Volatile Methyl Siloxanes in Vietnamese Micro-Environments
}

\author{
Hoang Quoc Anh ${ }^{1,2}$, Le Thi Hanh ${ }^{1}$, Dang Minh Huong Giang ${ }^{1}$, \\ Nguyen Thi Thanh Huyen ${ }^{1}$, Chu Ngoc Chau ${ }^{1}$, Phung Duc Hoa ${ }^{3}$, \\ Tu Binh Minh ${ }^{1}$, Tran Manh Tri ${ }^{1, *}$ \\ ${ }^{I}$ VNU University of Science, Vietnam National University, Hanoi, 19 Le Thanh Tong, Hanoi, Vietnam \\ ${ }^{2}$ Center of Advanced Technology for the Environment (CATE), Ehime University, \\ 3-5-7 Tarumi, Matsuyama 790-8566, Japan \\ ${ }^{3}$ Institute of Environmental Technology-Vietnam Academy of Science and Technology, \\ 18 Hoang Quoc Viet, Hanoi, Vietnam
}

Received 31 December 2019

Revised 19 February 2020; Accepted 03 March 2020

\begin{abstract}
In this study, the gas-particle distribution characteristics and emission sources of phthalate esters (PAEs) and volatile methyl siloxanes (VMSs) were evaluated for indoor air samples collected from different micro-environments such as homes, offices, kindergartens, hair salons, laboratories, and cars in 4 cities and provinces of Hanoi, Bac Ninh, Thai Binh, and Tuyen Quang, northern Vietnam. In general, total concentrations of PAEs and VMSs were higher in gas phase as compared to particle phase; however, phase distribution profiles of individual compounds were strongly related to their structures and physicochemical properties. For examples, low-molecularweight compounds such as dimethyl phthalate, diethyl phthalate, D3, D4, L4, and L5 were more abundant in gas phase, while heavier compounds like di(2-ethylhexyl) phthalate and L8 were preferentially associated with particle phase. Assessment of PAE emission sources is relatively difficult because they have been applied in different consumer products and materials. Significant correlation between cyclic VMSs (e.g., D4, D5, and D6) was observed, suggesting their applications in cosmetics and personal care products.
\end{abstract}

Keywords: Phthalate esters, volatile methyl siloxanes, indoor air, phase distribution, source apportionment.

\footnotetext{
* Corresponding author.

Email address: manhtri0908@gmail.com

https://doi.org/10.25073/2588-1140/vnunst.4985
} 


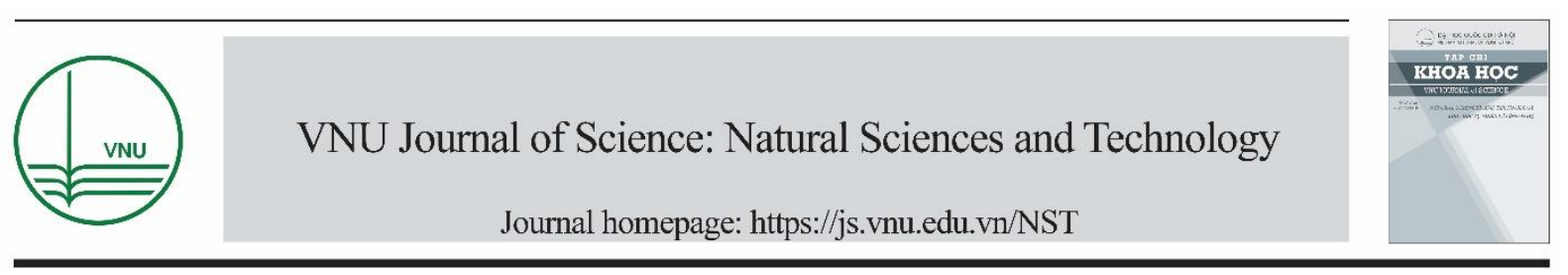

\title{
Đánh giá sự phân bố pha khí-hạt và nguồn gốc của các ester phthalate và methyl siloxane dễ bay hơi trong không khí trong nhà tại Việt Nam
}

\author{
Hoàng Quốc Anh ${ }^{1,2}$, Lê Thị Hạnh ${ }^{1}$, Đặng Minh Hương Giang ${ }^{1}$, \\ Nguyễn Thị Thanh Huyền ${ }^{1}$, Chu Ngọc Châu ${ }^{1}$, Phùng Đức Hòa ${ }^{3}$, \\ Từ Bình Minh ${ }^{1}$, Trần Mạnh Trí1,** \\ ${ }^{1}$ Truờng Đại học Khoa học Tự nhiên, Đại học Quốc gia Hà Nội, 19 Lê Thánh Tông, Hà Nội, Việt Nam \\ ${ }^{2}$ Trung tâm Công nghệ Tiên tiến cho Môi truờng (CATE), Đại học Ehime, 3-5-7 Tarumi, \\ Matsuyama 790-8566, Nhật Bản \\ ${ }^{3}$ Viện Công nghệ môi truờng-Viện Hàn lâm Khoa học và Công nghệ Việt Nam, \\ 18 Hoàng Quốc Việt, Hà Nội, Việt Nam \\ Nhận ngày 31 tháng 12 năm 2019 \\ Chỉnh sửa ngày 19 tháng 02 năm 2020; Chấp nhận đăng ngày 03 tháng 3 năm 2020
}

Tóm tắt: Trong nghiên cứu này, đặc trưng phân bố của các ester phthalate (PAEs) và methyl siloxane dễ bay hơi (VMSs) trong pha khí và pha hạt và nguồn gốc của chúng được đánh giá trong mẫu không khí từ các vi môi trường khác nhau như nhà ở, văn phòng, nhà trẻ, salon tóc, phòng thí nghiệm và ô tô tại 4 tỉnh, thành phố ở miền Bắc Việt Nam là Hà Nội, Bắc Ninh, Thái Bình và Tuyên Quang. Nhìn chung nồng độ tổng PAEs và VMSs trong pha khí cao hơn trong pha hạt, tuy nhiên đặc trưng cân bằng pha của từng chất có liên quan mật thiết đến cấu trúc phân tử và tính chất hóa lý của chúng. Ví dụ các hợp chất có phân tử khối thấp như dimethyl phthalate, diethyl phthalate, D3, D4, L4 và L5 phân bố chủ yếu trong pha khí, trong khi các chất có phân tử khối cao hơn như di(2ethylhexyl) phthalate và L8 lại tích lũy nhiều hơn trong pha hạt. Đánh giá nguồn phát thải của PAEs là một bài toán tương đối phức tạp do sự ứng dụng rộng rãi của chúng trong nhiều sản phẩm tiêu dùng và vật liệu khác nhau. Các chất VMSs mạch vòng (D4, D5 và D6) có mối tương quan khá rõ ràng, phản ánh ứng dụng chủ yếu của chúng trong các sản phẩm chăm sóc cá nhân.

Tù khóa: Ester phthalate, methyl siloxane dễ bay hơi, không khí trong nhà, phân bố pha, đánh giá nguồn gốc.

\footnotetext{
* Tác giả liên hệ.

Địa chỉ email: manhtri0908@gmail.com

https://doi.org/10.25073/2588-1140/vnunst.4985
} 


\section{Mở đầu}

Các ester phthalate (phthalic acid esters, PAEs) được bắt đầu tổng hợp trên quy mô công nghiệp từ những năm 1920 để làm chất dẻo hóa nhựa và đến những năm 1950 thì các hợp chất này đã trở nên phổ biến khi chúng được dùng trong công nghệ sản xuất nhựa polyvinyl chloride (PVC) [1]. Hiện nay, PAEs được áp dụng trong nhiều lĩnh vực công nghiệp và sản xuất khác nhau. Do tương tác của PAEs với polymer nền chỉ là tương tác vật lý nên các hợp chất này có thể phát tán từ vật liệu gốc ra môi trường xung quanh trong quá trình sản xuất, sử dụng và thải bỏ các vật liệu đó [2]. PAEs đã trở thành các chất ô nhiễm phổ biến trong nhiều thành phần môi trường khác nhau như không khí, bụi, nước, đất, trầm tích và sinh vật [3]. Một số PAEs được xác định là chất gây rối loạn nội tiết và độc tính của chúng đối với sức khỏe môi trường và sức khỏe con người là vấn đề được quan tâm nghiên cứu [3].

Các methyl siloxane dễ bay hơi (volatile methyl siloxanes, VMSs) được tổng hợp và áp dụng rộng rãi trong nhiều lĩnh vực công nghiệp, đặc biệt là công nghiệp sản xuất hàng tiêu dùng và sản phẩm chăm sóc cá nhân [4]. Các VMSs được phân biệt dựa trên cấu trúc và số nguyên tử silicon trong phân tử của chúng: VMSs mạch hở (linear VMSs, LVMSs, ví dụ như L4, L5, L6) và VMSs mạch vòng (cyclic VMSs, CVMSs, ví dụ như D4, D5, D6). Tương tự như các PAEs, VMSs cũng được phát hiện phổ biến trong nhiều thành phần môi trường khác nhau như không khí, bụi, nước, đất, trầm tích và bùn thải, đặc biệt là các không gian trong nhà $[5,6]$. Mặc dù các tác động tiêu cực của VMSs trên cơ thể người chưa được chứng minh một cách rõ ràng nhưng độc tính của các hợp chất này trên động vật thí nghiệm đã khiến cho nhiều nghiên cứu về sự ô nhiễm VMSs trong môi trường và phơi nhiễm trên cơ thể người được thực hiện trong thời gian gần đây [7-10].

Tại Việt Nam, các nghiên cứu về sự tồn tại của PAEs và VMSs trong môi trường không khí và bụi và tác động của chúng đối với con người chỉ mới được thực hiện trong vòng 5 năm trở lại đây [8-12]. Nồng độ của VMSs trong mẫu bụi trong nhà tại Việt Nam nhìn chung thấp hơn so với một số quốc gia khác trên thế giới như Hy Lạp, Kuwait, Hàn Quốc, Nhật Bản, Mỹ và Trung Quốc [8]. Một số nghiên cứu khác đã chỉ ra rằng nồng độ PAEs trong môi trường bụi và không khí tại khu vực đô thị lớn như Hà Nội cao hơn so với các khu vực có mức độ đô thị hóa và mật độ dân số thấp hơn ở miền Bắc và miền Trung nước ta $[12,13]$. Tuy nhiên, các nghiên cứu chuyên sâu về PAEs và $\mathrm{VMSs}$ trong môi trường không khí với số lượng mẫu lớn, phân tích đồng thời nồng độ trong pha khí và pha hạt cũng như khai thác triệt để kết quả phân tích để đánh giá nguồn gốc của chúng ở nước ta còn rất hạn chế. Trong nghiên cứu này, chúng tôi đánh giá đặc trưng phân bố ở 2 pha khí-hạt và nguồn gốc của 10 PAEs và 10 VMSs trong mẫu không khí trong nhà và trong ô tô lấy tại các không gian vi môi trường khác nhau tại 4 tỉnh, thành phố ở miền Bắc nước ta là Hà Nội, Bắc Ninh, Thái Bình và Tuyên Quang.

\section{2. Đối tượng và phương pháp nghiên cứu}

\subsection{Thông tin về mẫu phân tích}

Mẫu không khí được thu thập bằng thiết bị lấy mẫu khí chủ động lưu lượng thấp (lowvolume air sampler LP-7 $230 \mathrm{~V}$ pump kit, A.P. Buck Inc., Mỹ) trong thời gian 12 đến 24 giờ cho mỗi mẫu và tốc độ dòng $4 \mathrm{~L} / \mathrm{min}$. Pha hạt được tích lũy trên màng lọc sợi thạch anh (QFF) (MQA, Whatman, kích thước lỗ 2,2 $\mu \mathrm{m}$ ). Các chất trong pha hơi được hấp phụ bởi 2 cột xốp polyurethane (PUF) đặt nối tiếp (ORBO-1000, đường kính và chiều cao mỗi cột PUF là $2,2 \mathrm{~cm}$ và $7,6 \mathrm{~cm}$ ). Thể tích các mẫu không khí thu thập được nằm trong khoảng 2.9 đến $5.8 \mathrm{~m}^{3}$. Tổng cộng 97 mẫu không khí (bao gồm 97 mẫu PUF và 97 mẫu $\mathrm{QFF}$ ) đã được thu thập trong khoảng thời gian từ cuối năm 2016 đến đầu năm 2017 tại Hà Nội, Bắc Ninh, Thái Bình và Tuyên Quang trong các không gian vi môi trường là nhà ở, văn phòng, nhà trẻ, salon tóc, phòng thí nghiệm và ô tô. 


\subsection{Phưong pháp phân tích ester phthalate và methyl siloxane}

Trong nghiên cứu này, chúng tôi phân tích hàm lượng của 10 PAEs bao gồm: dimethyl phthalate (DMP), diethyl phthalate (DEP), dipropyl phthalate (DPP), diisobutyl phthalate (DiBP), dibutyl phthalate (DBP), di-n-hexyl phthalate (DnHP), benzyl butyl phthalate (BzBP), dicyclohexyl phthalate (DCHP), di(2ethylhexyl) phthalate (DEHP) và di-n-octyl phthalate (DnOP); và 10 VMSs bao gồm: D3, D4, D5, D6, L4, L5, L6, L7, L8 và L9. Các chất nội chuẩn deuterium hóa của PAEs bao gồm DMP-d4, DEP-d4, DPP-d4, DiBP-d4, DnHP-d4, BzBP-d4 và DEHP-d4. Chất nội chuẩn của các VMSs là tetrakis(trimethylsiloxy)-silane (M4Q). Các chất chuẩn và chất nội chuẩn cho phân tích PAEs và VMSs được cung cấp bởi SigmaAldrich và được chuẩn bị trong dung môi hexane.

Trước khi phân tích, mỗi mẫu PUF và QFF được thêm chuẩn chất nội chuẩn (500 ng mỗi chất PAEs-d4 và $200 \mathrm{ng}$ M4Q). Mẫu PUF được chiết lần lượt với $100 \mathrm{~mL}$ và $80 \mathrm{~mL}$ hỗn hợp dichloromethane $(\mathrm{DCM}) /$ hexane $(3: 2, \mathrm{v} / \mathrm{v})$ trên máy lắc orbital (Stuart, Nhật Bản), mỗi lần chiết trong $30 \mathrm{~min}$. Dịch chiết được cô đặc bằng bộ cô quay chân không và bộ thổi khí nitrogen đến thể tích $1 \mathrm{~mL}$ và chuyển vào $\mathrm{GC}$ vial. Mẫu $\mathrm{QFF}$ được chiết bằng kỹ thuật tương tự với hỗn hợp $\mathrm{DCM} /$ hexane $(3: 2, \mathrm{v} / \mathrm{v})$, chiết lặp 3 lần, mỗi lần với $5 \mathrm{~mL}$ dung môi trong $5 \mathrm{~min}$. Dịch chiết được cô đặc dưới dòng khí nitrogen đến thể tích $1 \mathrm{~mL}$ và chuyển vào $\mathrm{GC}$ vial. $\mathrm{PAEs}$ và $\mathrm{VMSs}$ được phân tích trên hệ thống sắc ký khí 7890B ghép nối khối phổ kế 5977A trên cột tách DB-5MS (chiều dài $30 \mathrm{~m}$, đường kính trong $0,25 \mathrm{~mm}$, bề dày lớp pha tĩnh $0,25 \mu \mathrm{m})$. Hệ thống phân tích và cột tách được cung cấp bởi Agilent Technologies. Các thông số về điều kiện tách và định lượng trên khối phổ cho các PAEs và VMSs được trình bày cụ thể trong các nghiên cứu trước đây của chúng tôi [9-11]. Độ thu hồi của các chất nội chuẩn và chất chuẩn thêm vào mẫu kiểm tra nằm trong khoảng từ 70 đến $120 \%$ với $\mathrm{RSD}<$ 20\%. Giới hạn phát hiện $(\mathrm{GHPH})$ của các PAEs nằm trong khoảng 0,5 đến $1,5 \mu \mathrm{g} / \mathrm{g}$ cho pha hạt và 0,03 đến $0,1 \mathrm{ng} / \mathrm{m}^{3}$ cho pha khí. GHPH của các VMSs dao động từ 1,5 đến $9 \mu \mathrm{g} / \mathrm{g}$ và 0,1 đến $0,7 \mathrm{ng} / \mathrm{m}^{3}$ cho pha hạt và pha khí, tương ứng.

\subsection{Phương pháp đánh giá nguồn gốc}

Toàn bộ tập số liệu về nồng độ 10 PAEs và 10 VMSs trong 97 mẫu PUF, 97 mẫu QFF và nồng độ tổng trong không khí được phân tích để tìm ra mối liên hệ giữa các chất phân tích và đánh giá nguồn gốc của chúng trong không khí. Phương pháp phân tích tương quan Pearson và phương pháp phân tích thành phần chính (principal component analysis PCA) đã được áp dụng. Đối với phân tích tương quan, hệ số tương quan lớn hơn 0,5 với giá trị $p<0,05$ được cho là có ý nghĩa thống kê. Đối với phân tích PCA, chúng tôi chỉ tập trung bàn luận đối với các thành phần $(\mathrm{PC})$ có phương sai lớn hơn 1 . Hệ số tương quan của các cấu tử trong mỗi PC có giá trị càng gần 1 chỉ ra mối liên hệ chặt chẽ của các cấu tử đó (ví dụ như có cùng nguồn gốc, phạm vi ứng dụng và/hoặc tính chất lý hóa tương tự). Số liệu được xử lý thống kê trên các phần mềm Microsoft Excel 2010 và Minitab 16.

\section{Kết quả và thảo luận}

\section{1. Đặc trưng cân bằng pha của ester phthalate}

Nồng độ tổng của PAEs (pha khí và pha hạt) trong các mẫu không khí nằm trong khoảng 110 đến $16000 \mathrm{ng} / \mathrm{m}^{3}\left(1000 \pm 1800 \mathrm{ng} / \mathrm{m}^{3}\right)$. Nồng độ tổng của PAEs trong pha khí và pha hạt lần lượt là $780 \pm 1700$ (khoảng 57-15000) và $270 \pm 200$ (khoảng 7,2-1100) ng/ $\mathrm{m}^{3}$. Nhìn chung, nồng độ tổng PAEs trong 2 pha phản ánh các hợp chất này tồn tại trong pha khí nhiều hơn so với pha hạt. Trong pha khí, nồng độ các chất giảm theo thứ tự DEP $>$ DiBP $>$ DBP $>$ DEHP $>$ DMP $>$ DCHP $>$ DPP $\approx$ BzBP $>$ DnOP $>$ DnHP. Trong pha hạt, chất có nồng độ cao nhất là $\mathrm{DEHP}$, tiếp theo là các chất DiBP $>$ DBP $>$ DCHP $>$ DEP > DnOP $>$ DPP $>$ BzBP $>$ DMP $>$ DnHP. Đặc trưng cân bằng pha khí-hạt của các $\mathrm{PAEs}$ được thể hiện trong Hình 1. 


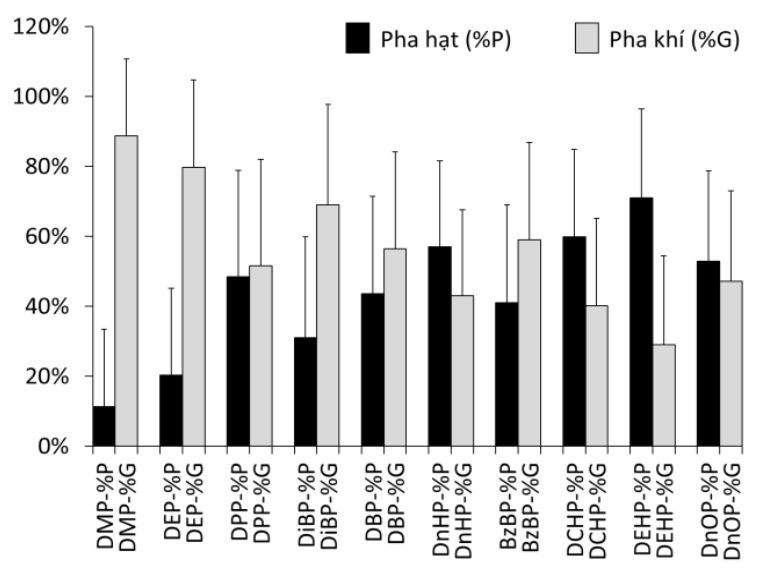

Hình 1. Đặc trưng cân bằng pha khí-hạt của các PAEs trong mẫu không khí trong nhà tại miền Bắc Việt Nam (trung bình và $\mathrm{SD}, n=97$ ).

Theo chiều tăng của phân tử khối, tỉ lệ của PAEs trong pha hạt có xu hướng tăng dần và tỉ lệ của chúng trong pha khí có xu hướng giảm dần. Ví dụ, tỉ lệ DMP trong pha khí và pha hạt lần lượt là $89 \pm 22 \%$ và $11 \pm 22 \%$, trong khi DEHP tồn tại chủ yếu trong pha hạt $(71 \pm 25 \%)$ so với pha khí ( $29 \pm 25 \%)$. Một số PAEs có phân tử khối trung bình như DBP và DnHP có tỉ lệ phân bố giữa 2 pha khá tương đồng. Các nghiên cứu trước đây cũng đã chỉ ra rằng, PAEs có phân tử khối thấp (ví dụ DMP đến DBP) tồn tại trong pha khí nhiều hơn trong pha hạt, trong khi các chất có phân tử khối lớn lại tích lũy chủ yếu trong pha hạt $[3,14]$. Kết quả thu được từ nghiên cứu này hoàn toàn phù hợp với các tính toán lý thuyết đối với một số hằng số cân bằng pha ví dụ như hệ số cân bằng khí-hạt $\left(\mathrm{K}_{\mathrm{P}}\right)$ và hệ số phân bố giữa octanol và không khí (KOA) [11].

\section{2. Đặc trung cân bằng pha của methyl siloxane}

Nồng độ tổng của VMSs (pha khí và pha hạt) trong các mẫu không khí có giá trị trung bình 470 \pm 430 (khoảng 42-1900) ng/ $\mathrm{m}^{3}$. Nồng độ VMSs trong pha khí $(320 \pm 320$, khoảng 24-1600 $\left.\mathrm{ng} / \mathrm{m}^{3}\right)$ nhìn chung cao hơn so với pha hạt (150 \pm 160, khoảng 13-880 ng/ $\mathrm{m}^{3}$ ). Nồng độ tổng của CVMSs (D3 đến D6) và LVMSs (L4 đến L9) tương đối đồng đều trong cả pha khí và pha hạt.
Trong cả 2 pha, nồng độ của các chất có xu hướng tăng theo chiều tăng phân tử khối, đặc biệt là trong pha hạt. Các chất có nồng độ cao nhất là D5, D6, L7 và L8.

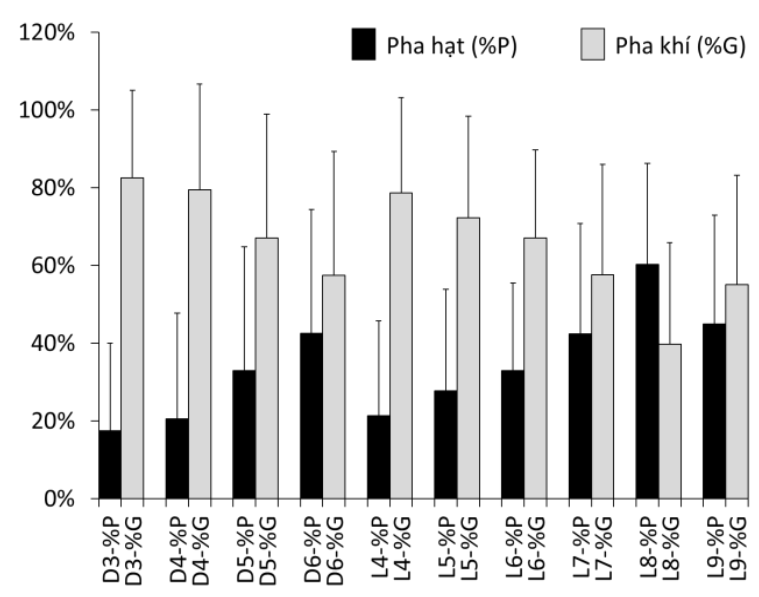

Hình 2. Đặc trưng cân bằng pha khí-hạt của các VMSs trong mẫu không khí trong nhà tại miền Bắc Việt Nam (trung bình và $\mathrm{SD}, n=97$ ).

Hình 2 thể hiện tỉ lệ của các VMSs giữa 2 pha khí và hạt. Ngoại trừ $\mathrm{L} 8$, các VMSs còn lại thể hiện xu hướng tồn tại trong pha khí nhiều hơn pha hạt, đặc biệt là các VMSs có phân tử khối thấp như D3 (83 $\pm 23 \%$ trong pha khí), D4 (79 \pm $27 \%)$ hay L4 (79 $\pm 24 \%)$. Các VMSs có phân tử khối cao có tỉ lệ trong pha hạt đáng kể, ví dụ như D6 (42 $\pm 32 \%$ trong pha hạt), L9 (45 $\pm 28 \%)$ và đặc biệt là L8 $(60 \pm 26 \%)$. Thông tin về cân bằng pha khí-hạt của VMSs trong không khí còn tương đối hạn chế do các nghiên cứu về chúng trước đây chủ yếu quan tâm đến pha khí. Vì vậy các số liệu về VMSs trong cả 2 pha khí và hạt của chúng tôi cung cấp những dữ liệu cơ bản và hoàn chỉnh về dự phân bố của VMSs trong không khí trong nhà tại Việt Nam. Đặc trưng tích lũy của VMSs trong pha hạt tương đối thống nhất với các mẫu không khí tại Albany, New York, Mỹ [7] và các mẫu bụi trong nhà thu thập tại Việt Nam và các nước khác trên thế giới, trong đó $\mathrm{D} 5$ và L8 là các hợp chất có nồng độ cao đáng kể [8]. 


\section{3. Đánh giá nguồn gốc của ester phthalate}

Kết quả phân tích PCA đối với tập số liệu nồng độ PAEs trong pha khí và pha hạt được trình bày trên Hình 3 . Trong pha hạt, 3 nhóm chất có mối liên hệ khá rõ ràng bao gồm: (DnHP, $\mathrm{BzBP}),(\mathrm{DCHP}, \mathrm{DEHP}, \mathrm{DnOP})$ và (DEP, DiBP, DBP). Trong pha khí, sự phân nhóm có những khác biệt nhất định so với pha hạt: (DEP, DiBP, $\mathrm{DBP}, \mathrm{DEHP}, \mathrm{DnOP})$ và (DPP, BzBP). Kết quả phân tích tương quan đã chỉ ra mối liên hệ đồng biến giữa (DnHP, BzBP) và (DCHP, DnOP) trong pha khí; cùng với $(\mathrm{DEP}, \mathrm{DBP})$ và (DPP, $\mathrm{BzBP}$ ) trong pha hạt. Các mối liên hệ này cho thấy, ngoại trừ trường hợp của $\mathrm{DiBP}$ và $\mathrm{DBP}$ là một cặp đồng phân với các tính chất hóa lý rất giống nhau nên có cùng phương thức tồn tại và vận chuyển trong môi trường, thì các mối liên hệ khác lại không phản ánh rõ vai trò của tính chất hóa lý đến sự phân nhóm PAEs.

Như vậy, cần phải xem xét đến yếu tố phạm vi và mức độ ứng dụng của các $\mathrm{PAEs}$ và ảnh hưởng của yếu tố này đến sự phân bố PAEs tại khu vực nghiên cứu. $\mathrm{DEP}$ và $\mathrm{DBP}$ là thành phần trong một số loại dung môi công nghiệp, dung môi trong nước hoa, chất kết dính, mực, chất diệt côn trùng, dược phẩm và mỹ phẩm [15]. Trong khi đó các $\mathrm{PAEs}$ như $\mathrm{DBP}, \mathrm{DEHP}, \mathrm{DCHP}$ và DnOP được dùng làm chất dẻo hóa trong công nghiệp polymer cũng như có mặt trong các sản phẩm nhựa và vật liệu xây dựng và nội thất ví dụ như các loại sơn và chất kết dính $[3,16,17]$. PAEs cũng có thể được phát thải từ những nguồn từ bên ngoài như phương tiện giao thông và vật liệu xây dựng của các cơ sở hạ tầng [18]. Mối tương quan đáng kể giữa $\mathrm{DEHP}, \mathrm{DCHP}, \mathrm{DBP}$ và $\mathrm{DiBP}$ cũng được phát hiện trong mẫu bụi trên mặt đường tại miền Bắc Việt Nam [19]. Mối quan hệ giữa $\mathrm{DnHP}$ và $\mathrm{BzBP}$ tuy được chỉ ra nhưng đây là các chất có nồng độ tương đối thấp với mức hàm lượng trong một số mẫu dưới giới hạn định lượng, do đó chúng tôi không bàn luận cụ thể về sự tương quan của 2 hợp chất này.
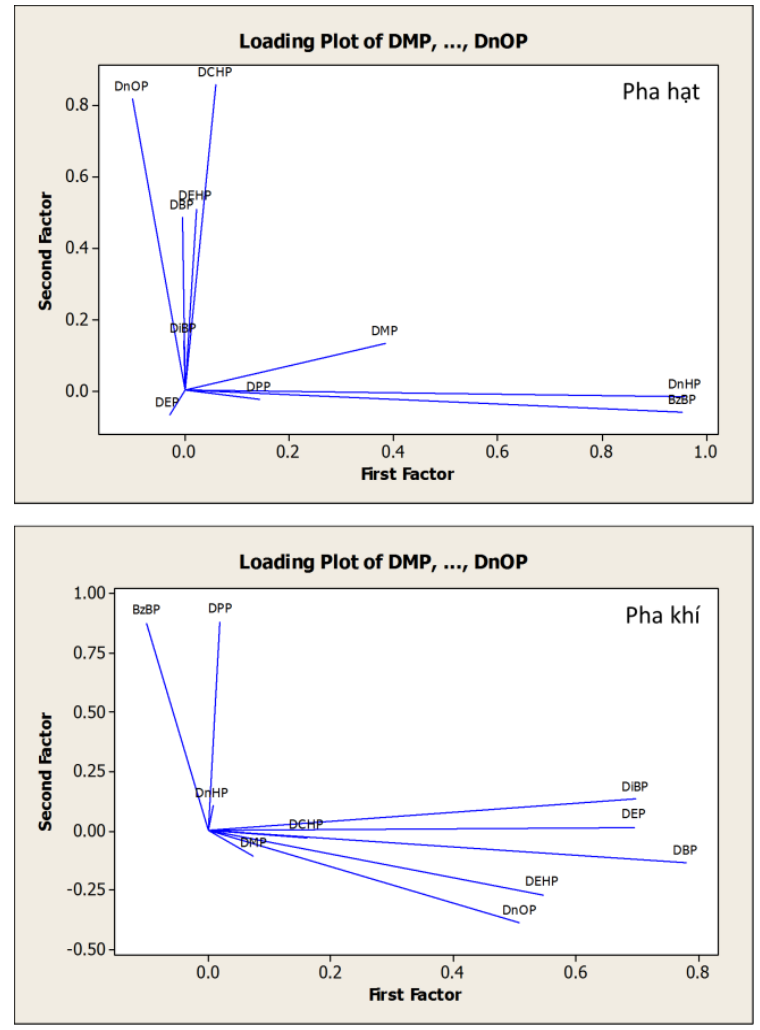

Hình 3. Kết quả phân tích PCA đối với nồng độ PAEs trong pha khí và pha hạt của mẫu không khí trong nhà tại miền Bắc Việt Nam.

Việc đánh giá nguồn phát thải của PAEs trong không khí trong nhà là tương đối phức tạp do một số PAEs là cấu tử chính trong các đối tượng môi trường (ví dụ như DBP) được ứng dụng rộng rãi trong nhiều lĩnh vực khác nhau. Hơn nữa, khi các vật liệu và sản phẩm có chứa PAEs cùng được sử dụng trong nhà thì rất khó để quy kết sự phát thải của từng PAEs ra môi trường không khí ứng với một nguồn cụ thể nào, đặc biệt là khi chúng ta không có số liệu đo đạc thực tế về nồng độ của các PAEs trong nguồn phát thải của chúng. Thêm vào đó, các nghiên cứu tổng thể hơn, quan tâm đến cả cân bằng giữa không khí và bụi lắng cũng như mối liên hệ giữa không khí trong nhà và ngoài trời là cần thiết để có thể đánh giá một cách đầy đủ và chính xác hơn về nguồn phát thải của PAEs. 


\section{4. Đánh giá nguồn gốc của methyl siloxane}

Kết quả phân tích tương quan và PCA đối với tập số liệu VMSs trong không khí đã chỉ ra mối liên hệ tương đối rõ ràng giữa các chất trong nhóm CVMSs (đặc biệt là D4, D5, D6 trong cả 2 pha khí và hạt) và trong nhóm LVMSs (đặc biệt là $L 8$ và $L 9$ trong pha hạt). Mặc dù sự tương quan của CVMSs và LVMSs là có ý nghĩa thống kê ( $p$ $<0,001)$ nhưng hệ số tương quan tương đối thấp ( $R=0,498$ và 0,356 cho pha hạt và pha khí, tương ứng). Các dữ liệu này góp phần đưa ra một giả thuyết rằng CVMSs và LVMSs cùng có nhiều ứng dụng trong không gian trong nhà nhưng trên các đối tượng khác nhau. CVMSs được tìm thấy chủ yếu trong các sản phẩm chăm sóc tóc, mỹ phẩm và dụng cụ nhà bếp tại Mỹ và Nhật Bản [20]; trong khi LVMSs lại có nồng độ cao hơn trong nhiều sản phẩm tiêu dùng khác nhau như sản phẩm chăm sóc cá nhân, đồ dùng cho trẻ em và các ứng dụng khác trên thị trường Trung Quốc [21,22]. Tại Việt Nam, hàm lượng của CVMSs (D4, D5 và D6) cũng đã được xác định trong các sản phẩm chăm sóc tóc $(8,77$ đến $515 \mu \mathrm{g} / \mathrm{g}$ ) và các sản phẩm này được đánh giá là nguồn phát thải chính của VMSs ra môi trường không khí và bụi tại các salon tóc ở Hà Nội [10]. Tuy nhiên, dữ liệu về sự tồn tại của VMSs, đặc biệt là các LVMSs trong các loại sản phẩm tiêu dùng khác nhau tại Việt Nam còn rất hạn chế, dẫn đến những khó khăn nhất định cho việc đánh giá nguồn phát thải của các chất ô nhiễm này.

3.5. Đánh giá so bộ về mối liên hệ giũa ester phthalate và methyl siloxane trong không khí

Theo hiểu biết của chúng tôi, hiện chưa có thông tin về sự tồn tại đồng thời và mối liên hệ của PAEs và VMSs trong không khí. Do đó, chúng tôi đã tiến hành một số phép phân tích và đánh giá thống kê sơ bộ đối với toàn bộ tập số liệu về 2 nhóm chất này trong mẫu không khí tại Việt Nam. Không có mối tương quan đáng kể giữa nồng độ tổng PAEs và VMSs trong không khí $(R=0,423, p<0,001)$. Tuy nhiên, kết quả phân tích PCA đã chỉ ra mối liên hệ giữa một số PAEs và $\mathrm{VMSs}$, ví dụ như $\mathrm{DEP}, \mathrm{D} 4, \mathrm{D} 5, \mathrm{D} 6$ và L8 trong pha hạt và $\mathrm{DEP}, \mathrm{DiBP}, \mathrm{D} 3, \mathrm{D} 4, \mathrm{D} 5$ và
D6 trong pha khí. Phương pháp phân tích tương quan cũng tiết lộ sự tương đồng giữa DEP và D5 trong pha hạt $(R=0,683, p<0,001)$ cũng như giữa DCHP và $\mathrm{L} 9$ trong pha khí $(R=0,615, p<$ $0,001)$. Mối liên hệ này có thể được giải thích bởi 2 nguyên nhân: i) các PAEs phân tử khối thấp (điển hình là $\mathrm{DEP}$ ) được dùng làm dung môi hoặc phụ gia trong các sản phẩm chăm sóc cá nhân có chứa VMSs, và ii) các PAEs phân tử khối cao (ví dụ như DEHP hay DCHP) có trong nhựa của vỏ chai chứa các sản phẩm chăm sóc cá nhân. Tuy nhiên, các giả thuyết này cần được kiểm chứng bằng số liệu đo đạc thực tế trong thời gian tới.

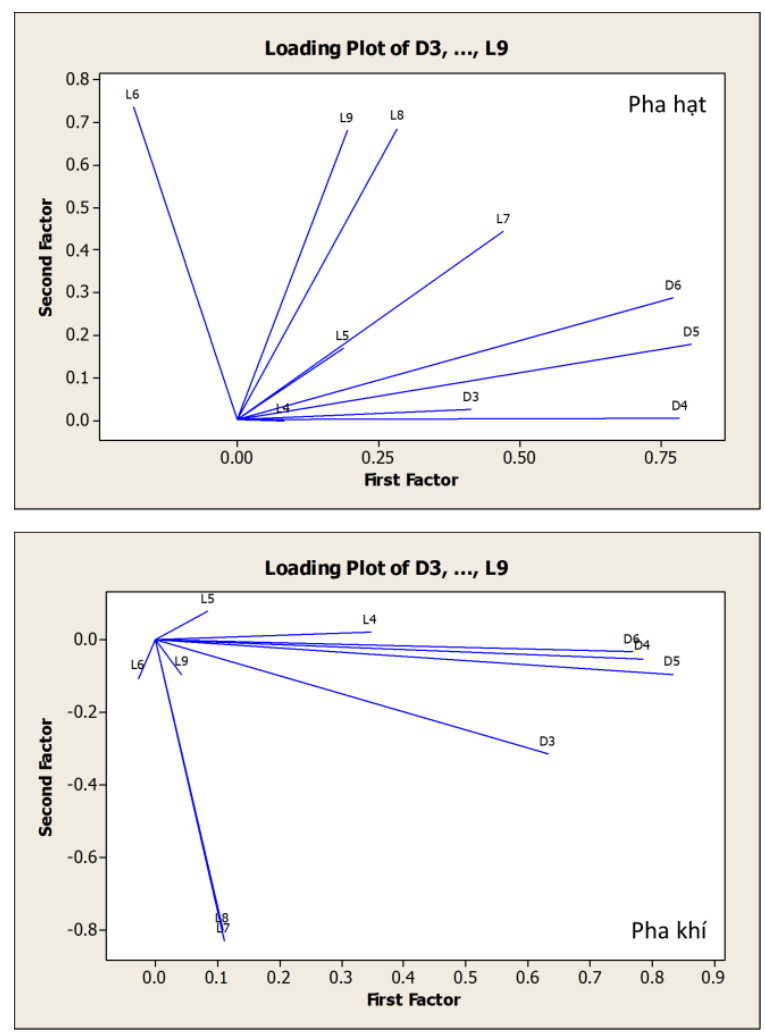

Hình 4. Kết quả phân tích PCA đối với nồng độ VMSs trong pha khí và pha hạt của mẫu không khí trong nhà tại miền Bắc Việt Nam.

\section{Kết luận}

Đặc trưng cân bằng pha khí-hạt của 10 PAEs, 4 CVMSs và 6 LVMSs được đánh giá 
trong gần 100 mẫu không khí trong nhà tại miền Bắc Việt Nam. Xu hướng phân bố của các chất nghiên cứu xác định bởi số liệu đo đạc thực tế hoàn toàn phù hợp với các tính toán lý thuyết. Theo đó, các hợp chất có phân tử khối thấp như DMP, DEP, D3, D4 và L4 tồn tại chủ yếu trong pha khí (trung bình khoảng $80 \%$ ), trong khi các hợp chất khó bay hơi hơn như DEHP hay L8 lại tích lũy mạnh trong pha hạt (trung bình khoảng 60 đến $70 \%$ ). Mối liên hệ giữa các chất nghiên cứu được đánh giá trong từng nhóm cũng như trên toàn bộ tập số liệu bằng phương pháp phân tích tương quan Pearson và phân tích thành phần chính PCA. Bên cạnh sự tương quan giữa các chất trong từng nhóm của chúng do nguồn gốc, ứng dụng và tính chất tương tự thì mối liên hệ giữa một số PAEs và VMSs cũng được chỉ ra. Dựa trên các dữ liệu sơ bộ này, việc thực hiện các nghiên cứu tổng thể hơn là rất cần thiết, ví dụ như đánh giá sự ô nhiễm PAEs và VMSs đồng thời trong môi trường trong nhà và ngoài trời cũng như các sản phẩm tiêu dùng khác nhau trên thị trường ở nước ta.

\section{Lời cảm ơn}

Nghiên cứu này được tài trợ bởi Quỹ Phát triển Khoa học và Công nghệ Quốc gia (NAFOSTED) trong đề tài mã số 104.012018.314.

\section{Tài liệu tham khảo}

[1] I. Kimber, J.R. Dearman, An assessment of the ability of phthalates to influence immune and allergic responses. Toxicology 271(3) (2010) 73-82. https://doi.org/10.1016/j.tox.2010.03.020.

[2] C. Christia, G. Poma, S. Harrad, C.A. de Wit, Y. Sjostrom, P. Leonards, M. Lamoree, A. Covaci, Occurrence of legacy and alternative plasticizers in indoor dust from various EU countries and implications for human exposure via dust ingestion and dermal absorption. Environ. Res. 171 (2019) 204-212. https://doi.org/10.1016/j. envres.2018.11.034.

[3] S. Net, R. Sempéré, A. Delmont, A. Paluselli, B. Ouddane, Occurrence, fate, behavior and ecotoxicological state of phthalates in different environmental matrices. Environ. Sci. Technol. 49(7) (2015) 4019-4035. https://doi.org/10.1021/ es505233b.

[4] K. Mojsiewicz-Pienkowska, D. Krenczkowska, Evolution of consciouness of exposure to siloxanes-review of publications. Chemosphere 191 (2018) 204-217. https://doi.org/10.1016/j. chemosphere.2017.10.045.

[5] T.M. Tran, A.Q. Hoang, S.T. Le, T.B. Minh, K. Kannan, A review of contamination status, emission sources, and human exposure to volatile methyl siloxanes (VMSs) in indoor environments. Sci. Total Environ. 691 (2019) 584-594. https://doi.org/10.1016/j.scitotenv.2019.07.168.

[6] K. Gaj, A. Pakuluk, Volatile methyl siloxanes as potential hazardous air pollutants. Pol. J. Environ. Stud. 24(3) (2015) 937-943. https://doi.org/10. $15244 /$ pjoes/34668.

[7] T.M. Tran, K. Kannan, Occurrence of cyclic and linear siloxanes in indoor air from Albany, New York, USA, and its implications for inhalation exposure. Sci. Total Environ. 511 (2015) 138-144. https://doi.org/10.1016/j.scitotenv.2014.12.022.

[8] T.M. Tran, K.O. Abualnaja, G.A. Asimakopoulos, A. Covaci, B. Gevao, B. Johnson-Restrepo, A.T. Kumosani, G. Malarvannan, T.B. Minh, B.H. Moon, H. Nakata, K.R. Sinha, K. Kannan, A survey of cyclic and linear siloxanes in indoor dust and their implications for human exposures in twelve countries. Environ. Int. 78 (2015) 39-44. https://doi.org/10.1016/j.envint.2015.02.011.

[9] T.M. Tran, H.T. Le, N.D. Vu, G.H.M. Dang, T.B. Minh, K. Kannan, Cyclic and linear siloxanes in indoor air from several Northern cities in Vietnam: levels, spatial distribution and human exposure. Chemosphere 184 (2017) 1117-1124. https://doi. org/10.1016/j.chemosphere.2017.06.092.

[10] T.M. Tran, T.B. Minh, N.D. Vu, Cyclic siloxanes in indoor environments from hair salons in Hanoi, Vietnam: emission sources, spatial distribution, and implications for human exposure. Chemosphere 212 (2018) 330-336. https://doi. org/10.1016/j.chemosphere.2018.08.101.

[11] T.M. Tran, H.T. Le, T.B. Minh, K. Kannan, Occurrence of phthalate diesters in indoor air from several Northern cities in Vietnam, and its implication for human exposure. Sci. Total Environ. 601-602 (2018) 1695-1701. https://doi. org/10.1016/j.scitotenv.2017.06.016.

[12] T.M. Tran, T.B. Minh, T.A. Kumosani, K. Kannan, Occurrence of phthalate diesters (phthalates), p-hydroxybenzoic acid esters (parabens), bisphenol A diglycidyl ether (BADGE) and their derivatives in indoor dust 
from Vietnam: Implications for exposure. Chemosphere 144 (2016) 1553-1559. https://doi. org/10.1016/j.chemosphere.2015.10.028.

[13] H.Q. Anh, K. Tomioka, N.M. Tue, L.H. Tuyen, N.K. Chi, T.B. Minh, P.H. Viet, S. Takahashi, A preliminary investigation of 942 organic micropollutants in the atmosphere in waste processing and urban areas, northern Vietnam: Levels, potential sources, and risk assessment. Ecotoxicol. Environ. Saf. 167 (2019) 354-364. https://doi.org/ 10.1016/j.ecoenv.2018.10.026.

[14] X. Wang, W. Tao, Y. Xu, J. Feng, F. Wang, Indoor phthalate concentration and exposure in residential and office buildings in Xi'an, China. Atmos. Environ. 87 (2014) 146-152. https://doi. org/10.1016/j.atmosenv.2014.01.018.

[15] D. Koniecki, R. Wang, R.P. Moody, J. Zhu, Phthalates in cosmetic and personal care products: concentrations and possible dermal exposure. Environ. Res. 111(3) (2011) 329-336. https://doi. org/10.1016/j.envres.2011.01.013.

[16] J. Zhao, Y. Ji, Z. Zhu, W. Zhang, L. Zhang, J. Zhao, PAEs occurrence and sources in road dust and soil in/around parks in May in Tianjin, China. Ecotoxicol. Environ. Saf. 147 (2018) 238-244. https://doi.org/10.1016/j.ecoenv.2017.08.014.

[17] T. Otake, J. Yoshinaga, Y. Yanagisawa, Exposure to phthalate esters from indoor environment. J. Expo. Sci. Environ. Epidemiol. 14 (2004) 524528. https://www.nature.com/articles/7500352.
[18] A. Markiewicz, K. Bjorklund, E. Eriksson, Y. Kalmykova, A.M. Stromvall, A. Siopi, Emissions of organic pollutants from traffic and roads: priority pollutants selection and substance flow analysis. Sci. Total Environ. 580 (2017) 1162-1174. https://doi.org/10.1016/j.scitotenv.2016.12.074.

[19] H.Q. Anh, T.M. Tran, N.T.T. Thuy, T.B. Minh, S. Takahashi, Screening analysis of organic micropollutants in road dusts from some areas in northern Vietnam: A preliminary investigation on contamination status, potential sources, human exposure, and ecological risk. Chemosphere 224 (2019) 428-436. https://doi.org/10.1016/j. chemosphere. 2019.02.177.

[20] Y. Horii, K. Kannan, Survey of organosilicone compounds, including cyclic and linear siloxanes, in personal-care and household products. Arch. Environ. Contam. Toxicol. 55 (2008) 701-710. https://doi.org/10.1007/s00244-008-9172-z.

[21] L. Xu, Y. Shi, N. Liu, Y. Cai, Methyl siloxanes in environmental matrices and human/fat from both general industries and residential areas in China. Sci. Total Environ. 505 (2015) 454-463. https:// doi.org/10.1016/j.scitotenv.2014.10.039.

[22] L. Xu, L. Zhi, Y. Cai, Methylsiloxanes in children silicone-containing products from China: profiles, leaching, and children exposure. Environ. Int. 101 (2017) 165-172. https://doi.org/10.1016/j.envint. 2017.01.022. 